\title{
Dynamic Tension Measurement System for Wire Rope of a Ultra-deep Multi Rope Winding Hoist
}

\author{
Wei Ma ${ }^{1,}$, Jianwei Wang ${ }^{1, b}$ and Jishun $\mathrm{Li}^{1,2, \mathrm{c}}$ \\ ${ }^{1}$ College of Mechatronics Engineering, Henan University of Science and Technology, Luoyang \\ 471003, China \\ ${ }^{2}$ Henan Key Laboratory of Mechanical Design and Transmission System, Luoyang \\ 471003, China \\ amawei-mail@163.com, bwjwzxl@foxmail.com, 9ljs_homework@163.com
}

\begin{abstract}
Keywords: Multi rope winding hoist, Wire rope, Dynamic tension, Real time monitoring, LabVIEW. Abstract. The dynamic tension is difficult to be measured, but it's very influential of ultra-deep multi rope winding hoist during the working process, the wire rope dynamic tension signal detection system of multi rope winding was designed by the virtual instrument. With the maturity of the technology, LabVIEW has been widely used in the field of industrial measurement, we apply it to the design of the dynamic tension signal detection system of the hoist, which make it detects the dynamic tension of wire rope in real time, and it also has the functions of parameter display, data storage, overlimit alarm and report printing. The operation of the measuring system is simple and efficient, which ensure the safety operation of ultra-deep multi rope winding hoist.
\end{abstract}

\section{Introduction}

Mine hoist is one of the important and critical equipment in the mine production, which is an important means of transportation to contact the aboveground and underground, as the "throat" equipment, it has an important status and function[1]. With the rapid development of our economy, a great deal of coal, metals and other resources are needed, because of the shallow--burial natural resources has failed to meet the needs of social development, so the Ultra-deep mine hoist should be built and will be become the future of the mining[2]. In the actual production process, wire rope is the easiest to have problems in hoisting system, and as the mine depth increases, the operating conditions are even worse, so it is much more important to detect the dynamic tension of the wire rope which connect the cage and the drum equipment.

At home and abroad, accidents caused by the lift container to fall happen almost every year, the falling is due to overload or uneven tension of wire rope, which cause significant economic and human losses, so the problem of the tension detection of wire rope has already become the consensus of us[3].The staff used the vibration wave method, the labeling method, the suspension method and the pressure round applanation tonometry method to measure the static measurements in the early[4]. These methods are simple and easy to operate, but they are low in accuracy and are not be monitored in real time, and it cannot really reflect the dynamic tension and its history of the wire rope in the process of ascension. This paper adopt virtual instruments to design a dynamic tension signal detection system of hoist, which combined with signal acquisition and processing. With LabVIEW's excellent measurements and friendly interface display[5],the system can detect the dynamic tension of the wire rope in real time, and it also has the functions of parameter display, data storage, overlimit alarm and report printing.

\section{Measurement System Composition}

In this paper, the object of the study is ultra-deep multi rope winding hoist, the diagram is shown in figure 1 . The wire rope dynamic tension detection device of the ultra deep mine hoist is a system that based on LabVIEW, the composition is shown in figure 2. The entire detection system consists of the host computer, hypogynous - machine and the machine interface at the central control room. When the elevator starts working, the staff issue an order that "start collecting" to the host machine through 
the machine interface at the central control room, the upper machine sent instructions to the lower machine via the wireless communication module, then the latter began to collect the tension data, and the tension data collected from the mine is sent to the top machine through wireless transmission, the top machine then transfers the received data to the PC for storage at last, at the same time, the visual expression of data and waveform is displayed on the main interface of LabVIEW edit control and display.

In order to meet the sensor installation convenient, not change the structure of the suspension system of the ultra-deep multi rope winding host and higher accuracy tension dynamic signal, the installation location of the sensor is shown in figure 3. The pressure sensors should be installed near the wire rope and cage joints, using the "buckle" link form, to turn the pull signal into the pressure signal[6]. And then after the transmitter conditioning module, the collected signals are amplified and filtered, demodulated and decrypted, and finally the stable output signal is obtained.

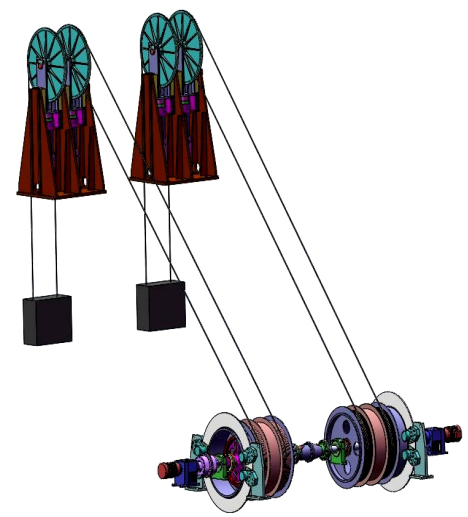

Fig. 1 Multi rope winding hoist diagram

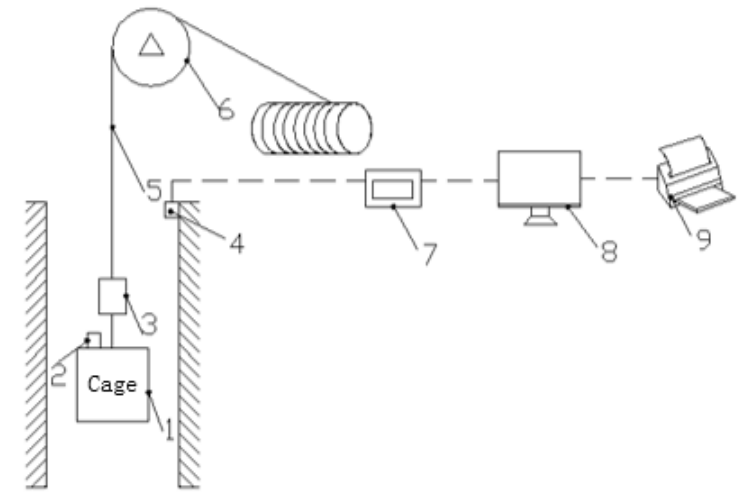

Fig. 2 Wire rope tension detection system schematic 1.Cage 2.Wireless data transceiver module 3.Tension sensor 4.Wireless data transceiver module 5.Wirerope 6.Head sheave 7.Winding drum 8.Transmitter 9.Embedded pc 10.Printer

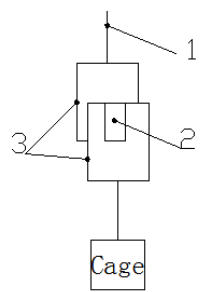

Fig. 3 Sensor installation schematic 1.Wire Rope 2.Pull pressure sensor 3."Buckle" connecting device

\section{Hardware Components of the Measurement System}

The hardware of the wire rope tension detection system is mainly composed of tension sensor, transmitter, data collection card, wireless communication module and embedded PC. The composition is shown in figure 4. 


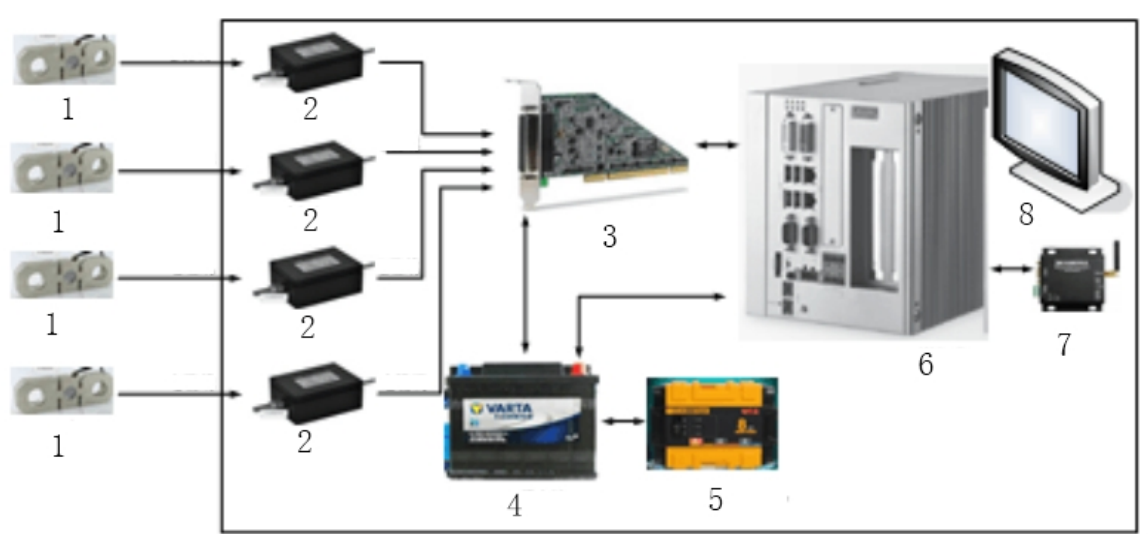

Fig. 4 Wire rope tension measuring devices structure schematic diagram 1.Sensors and connecting devices 2.Transmitter 3.PCI-9221 DAQ 4.12V80AH battery 5.NFA charger 6.UNO-3082 embedded PC 7.Wireless communication module 8.PC

\section{Sensor Design.}

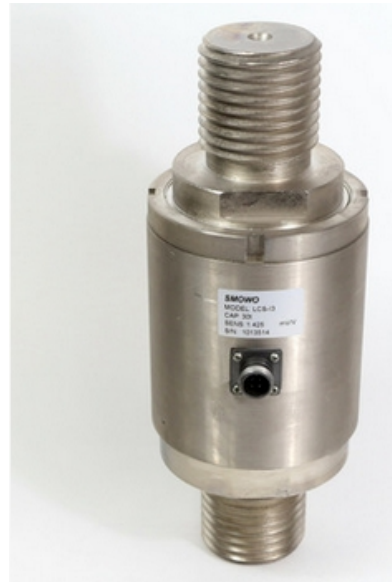

Fig. 5 Tension-compression sensor

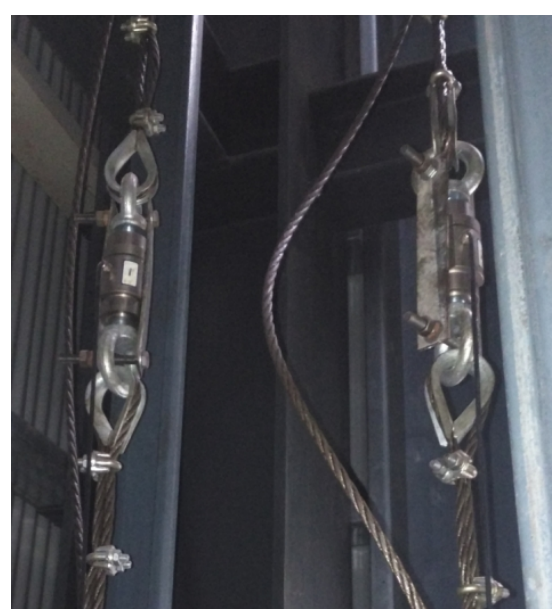

Fig. 6 Sensor job site connection diagram

Because of special condition environment of the Ultra-deep Mine, so the requirements for the sensor are higher[7]. In addition, the strain detection system is used to measure the wire rope tension and its change in the process of ascension, higher accuracy and sensitivity of the sensor and payload up to 50t, so the 200t column tension sensor is adopted, as shown in figure 5.This type of sensor has many advantages, such as high precision, reliable performance, dust-protection seal and wide range, we can place sensors inside two connected devices via the "buckle" form, the purpose of this is converted the dynamic tension signal of the wire rope into pressure signal at work[8], the job site connection is shown in figure 6.There are three reasons for this: firstly, the tension signal measurement is more timely and the error is smaller; secondly, the sensor cann't connect directly to the wire rope to prevent serious damage if the sensor fails; thirdly, it can protect the sensors and convenient disassembly to extend its life span.

\section{Transmitter Select}

The sensor's pull signal should be converted into an effective electrical signal, amplified and filtered at the same time, then the data can be received and stored by the PCI-9221.The traditional transmitter structure function is single, which can only convert analog signals into electrical signals, in the case of high signal strength needed conditions, the signal conditioning module should be added. This test system choose the EMGZ310 transmitter, not only the pull pressure signal can be converted into standard electrical signals for the back-end control system, but also can provide stable excitation voltage to the sensor, signal filter, signal amplification, automatic tension zero calculation and other functions, optimized the detection system. 


\section{Data Acquisition Card Select}

The data acquisition card is the core module of the hardware of the wire rope tension detection system. It is used primarily to take a non-electric or electrical signal automatically from sensors and other analog and digital devices, and then send to the host computer for the analysis processing [9].The new generation high performance DAQ card--PCI-9221 was chosen for the strain detection system. This product has multi channel input and output, programmable function of $\mathrm{I} / \mathrm{O}$, a number of operations at the same time, running full function. Compared with similar products, superior performance, high accuracy, strong stability, can ensure the accuracy of data collection in the case of high sampling rate.

\section{UNO-3082 embedded PC}

The UNO-3082 is a high-performance embedded industrial-level controller, it can connect three monitors and four PCI extensions, at the same time, in order to connect machine visual application conveniently, it equip with two IEEE -1394 interfaces.

\section{Software Design of Detection System}

The software design of the dynamic tension detection system of the ultra-deep mine hoist is based on Hierarchical design idea of LabVIEW, The software module is clear in structure, simple in operation interface and convenient for later maintenance and optimization. The problems due to the modification and debugging of the source code of the traditional industrial control software is avoided,which include the long development cycle, the tedious task, the low repetition rate, the high cost, the low accuracy and the poor real-time performance and so on[10]. The display interface of the detection system is shown in Figure 7, and the corresponding program block diagram is shown in Figure 8.

The main interface is the main body of the measurement system, which have the function of graphics and data display, in addition to the Capture button, stop button and the upper limit of tension adjustment command button.

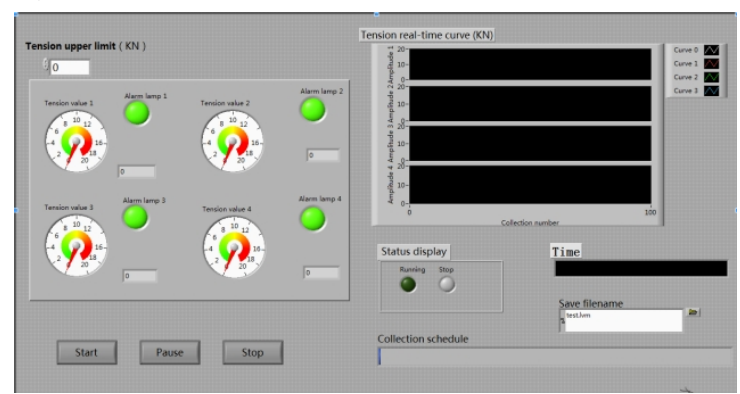

Fig. 7 Display interface of detection system

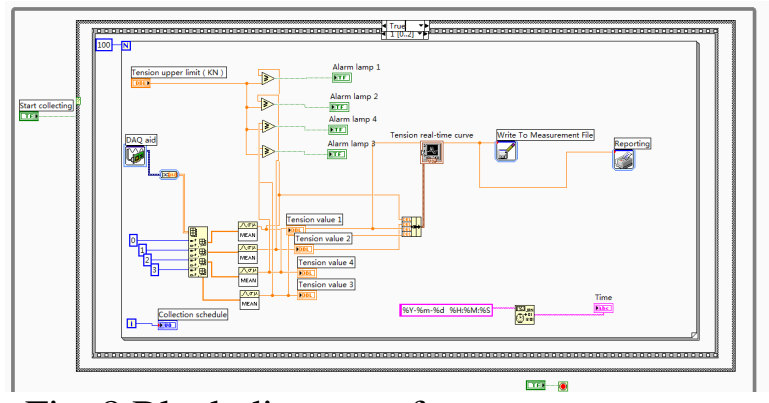

Fig. 8 Block diagram of measurement system

When the lifting system is running, click the Capture button to begin collecting, the detection system begins to work, collect tension values are shown in the main interface of the testing system, and if the collected tension value exceeds the warning value set, for the sake of safety in production, the alarm light will change from green to red.

The tension value collected by the system will be displayed at the same time in the waveform and tension indicator box, and will be written to the file which has been set up to facilitate the late problems, the historical data for analysis and print statements. The system can be monitored on-line in real time, which can reflect the dynamic tension of wire rope and the course of change. In the digital processing stage, the filter can be used to filter the collected digital signals, and get more reliable data. Therefore, the system can meet the requirements of mine safety production, and record the data, the long term work records provide data support for safety production. 


\section{Conclusion}

(1) The paper puts forward the use of interlocking "buckle" structure, by which the tension are converted into pressure signal ,so it is able to get more seasonable accurate signal and ensure the safety of production at the same time.

(2)The measurement system which is based on LabVIEW realize the real-time monitoring of the dynamic tension detection system of the ultra deep mine hoist, the system can realize the online storage and printing of the data report, and provide the alarm function. The measurement system is simple, fast and efficient, the system detects security risks timely when it is running by which the protection of personnel and equipment safety can be guaranteed. So it improves the safety and stability of the operation of the super deep well hoist.

\section{Acknowledgments}

The research work was supported by National 973 Program of China (Grant No. 2014CB049402), Project of Basic and Advanced Technology Research of Henan Province of China (No. 132300410001), Fund Project of Science and Technology Research Key Project of Henan Provincial Education Department of China (No. 14B460016), Program for Innovative Research Team (in Science and Technology) in University of Henan Province (No. 15IRTSTHN008).

\section{References}

[1] Hong Nie. Development and current situation of mine hoist[J]. Mining Machinery,2015.43 (8): 13-17.

[2] Ji-Wen Teng, Xue-mei Zhang, Hui Yang. China main energy--Second deep space exploration, development and efficient utilization of coal[J]. Progress in Geophysics, 2008.23 (8): 972-992.

[3] Chun-jing Li, Ji-wen Tan, Jun Tian. Research status and trend of wire rope tension detection [J]. Coal Mine Safety,2006.01:53-55.

[4] Ling Weng. Computer simulation of hoisting rope tension[J]. Coal Mine Machinery, 2013.12:224-226.

[5] Xi-hui Chen, Yin-hong Zhang. LabVIEW 8.20 program design from beginning to mastery[M]. Beijing: Tsinghua University Press,2007.60-80.

[6] Xu-ge Lu, Yun-xiao Fan, Ying Wu. The dynamic monitoring system design of hoisting machine wire rope[J]. Colliery Mechanical \& Electrical Technology, 2007.03:8-10.

[7] Hao-liang Feng, Wei Ma, Ji-shun Li. Dynamic response analysis for wire rope tension balance device of a ultra-deep multi rope winding hoist[J]. Journal of Henan University of Science \& Technology(Natural Science),2016.37(1):9-14.

[8] Shou-bo Lu, Guo-kai Zhang. Design and realization of steel wire rope tension tester based on LabWindows/CVI[J]. Computer Measurement and Control,2015.23 (8):2924-2927.

[9] Ying Wang. Belt conveyor strain detection system study based on the Virtual instrument[D]. Huainan: Anhui University of Science and Technology,2011.

[10] Wei-bo Li, Xia Hu. Design of monitoring system for mine hoist based on LabVIEW[J].Coal Mine Machinery,2015.36(9):32-34. 